\title{
Factores de riesgo familiares y prácticas de disciplina severa que predicen la conducta agresiva infantil
}

\author{
Family risk factors and harsh discipline practices that predict child's aggressive behavior
}

\author{
Juan Jiménez Flores, ${ }^{\mathrm{a}, *}$, Luz María Flores Herrera ${ }^{\mathrm{a}}$, César Merino-Soto ${ }^{\mathrm{b}}$ \\ ${ }^{a}$ Facultad de Estudios Superiores Zaragoza, Universidad Nacional Autónoma de México, México \\ bUniversidad de San Martín de Porres, Perú
}

\section{Resumen}

Objetivos: Se evaluó empíricamente un modelo de factores de riesgo familiares y prácticas de disciplina severa que predicen la conducta agresiva infantil, identificando su adecuación en términos del ajuste de datos y congruencia conceptual. Método: Se utilizó una muestra de 409 madres de niños de escuelas primarias públicas. Los factores de riesgo familiares seleccionados fueron: síntomas de ansiedad materna, violencia marital y prácticas de disciplina severa; la variable criterio fue la conducta agresiva infantil. El modelo supone que la ansiedad materna y la violencia marital tienen efectos sobre la conducta agresiva infantil, mediados por las prácticas de disciplina severa. La estrategia de investigación se diseñó con un modelo de ecuaciones estructurales, el cual fue satisfactorio (CFI $=.952$, NNFI $=.94$ y RMSEA $=$ .08 ); el modelo predijo el $34 \%$ de la varianza de los problemas de conducta agresiva infantil. Por sus efectos directos, la disciplina severa depende de la ansiedad materna $(\beta=.44)$ y de la violencia marital $(\beta=.10)$. La disciplina severa es una variable mediadora entre la conducta agresiva de los niños y la ansiedad materna y la violencia marital $(\beta=.59)$. Resultados: Los resultados indican que la ansiedad materna y la violencia marital tienen efectos significativos en los problemas de conducta agresiva de los niños mediados por las prácticas disciplinarias. Conclusión: La confirmación de los resultados requiere de nuevos estudios con diferentes poblaciones que permitan generalizar los hallazgos.

Palabras clave: modelamiento de ecuaciones estructurales, agresividad, niños, crianza parental, violencia familiar.

Para citar este artículo:

Jiménez, J., Flores, L. M., \& Merino-Soto, C. (2019). Factores de riesgo familiares y prácticas de disciplina severa que predicen la conducta agresiva infantil. Liberabit, 25(2), 195-212. https://doi.org/10.24265/ liberabit.2019.v25n2.05

\begin{abstract}
Objective: A model of family risk factors and harsh discipline practices that predict child's aggressive behavior was empirically evaluated, identifying its adequacy in terms of data adjustment and conceptual congruence. Method: A sample of 409 mothers of children from public elementary schools was used. The selected family risk factors were: symptoms of maternal anxiety, marital violence and harsh discipline practices; the criterion variable was the child's aggressive behavior. The model assumes that maternal anxiety and marital violence have effects on child's aggressive behavior, which is mediated by harsh discipline practices. The research strategy was designed with a structural equation model. The results were satisfactory $(\mathrm{CFI}=.952$, NNFI $=.94$ and RMSEA $=.08$ ) and the model predicted $34 \%$ of the variance of child's aggressive behavior problems. Due to its direct effects, harsh discipline depends on maternal anxiety $(\beta=.44)$ and marital violence $(\beta=.10)$. Harsh discipline is a mediating variable between child's aggressive behavior and maternal anxiety and marital violence ( $\beta=.59$ ). Results: The results indicate that maternal anxiety and marital violence have significant effects on child's aggressive behavior problems, which are mediated by disciplinary practices. Conclusion: The results must be confirmed with new studies conducted in different populations that allow generalizing the findings.
\end{abstract}

Keywords: structural equation modeling, aggressiveness, children, parenting, family violence.

Este es un artículo Open Access bajo la licencia Creative Commons Atribución-NoComercial-CompartirIgual 4.0

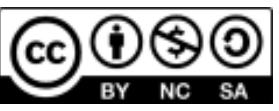




\section{Introducción}

El comportamiento agresivo infantil es uno de los grandes problemas de salud mental que afecta la integridad psicológica y las relaciones sociales de los niños; existen evidencias que asocian el comportamiento agresivo de los niños con factores familiares de riesgo que ocurren durante el desarrollo infantil (Flores, Villegas, Martínez, \& Palacios, 2017; Sánchez, 2018). En la actualidad su solución se ha concretado en una demanda social que lleva a que los expertos realicen esfuerzos para prevenir y controlar sus consecuencias negativas (Cova et al., 2019). Se ha observado que la conducta agresiva en la infancia persiste hasta llegar a la edad adulta y se asocia con otros trastornos psiquiátricos (Durand, Barlow, \& Pecina-Hernández, 2007; Haan, Prinzie, \& Dekoviæ, 2010) y comportamientos de violencia y conducta antisocial los cuales tienden a estabilizarse con el tiempo (Flores, Ruíz, Jiménez, \& Mercado, 2017; Silva, 2003).

La agresividad es un fenómeno caracterizado por conductas dirigidas a ocasionar daño a otras personas, animales, objetos o bien, a sí mismo (Fajardo \& Hernández, 2008). Las primeras manifestaciones de comportamiento agresivo surgen en la infancia temprana: los niños aprenden patrones de comportamiento agresivo como una manera de relacionarse con los demás (Berkowitz, 2008). La agresión es resultado del aprendizaje y las condiciones ambientales que afectan la habilidad para regular las emociones y la conducta (Ferguson, Cassells, MacAllister, \& Evans, 2013).

Los psicólogos observan con frecuencia que los niños agresivos provienen de familias violentas en que predomina un ambiente emocional negativo entre los padres. Con base en los resultados de estudios se ha logrado identificar factores de riesgo familiares asociados con la agresividad infantil, los cuales se pueden clasificar como: a) síntomas de ansiedad en las madres (Jiménez-Flores, 2014), b) violencia marital (Rasit \& Songul, 2013) y c) prácticas de disciplina severa (Chang, Dodge, Schwartz, \& McBride-Chang, 2003; Jiménez, 2014).

Los factores de riesgo familiares pueden ser distales o proximales, los primeros promueven problemas de ajuste social; se ha señalado que los conflictos maritales y los problemas emocionales de los padres son factores de riesgo familiares distales (Reid \& Patterson, 1989). Estos determinantes distales influyen en el desarrollo del comportamiento agresivo hasta que los padres interactúan con sus hijos durante su vida diaria, particularmente, en el área de la disciplina. Las prácticas disciplinarias de los padres pueden llegar a constituirse como factores de riesgo familiares proximales (Jiménez, 2014).

Por consiguiente, la psicopatología parental, el clima emocional familiar y las deficiencias parentales modelan las conductas que en el futuro dificultarán la integración adecuada del niño en la escuela y en la sociedad (Benzies, Harrison, \& Magill-Evans, 2004; Langrock, Compas, Keller, Merchant, \& Copeland, 2002; MacPhee \& Andrews, 2006; Sánchez, 2018; Sander \& McCarty, 2005).

La conducta agresiva infantil frecuentemente se asocia con síntomas de ansiedad en las madres de estos niños (Ginsburg, Grover, \& Ialongo, 2004). Las madres ansiosas suelen ser agresivas y generan un ambiente familiar conflictivo dando lugar a graves desajustes emocionales y conductuales en sus hijos (Jaffe, Hanscombe, Haworth, Davis, \& Plomin, 2012). Por esta razón, los problemas de ansiedad materna son factores de riesgo familiares que deben ser identificados y controlados por los profesionales del comportamiento (Reid \& Patterson, 1989; Reppeti, Taylor, \& Seeman, 2002; Scaramella \& Leve, 2004).

La ansiedad es una emoción compleja, difusa y desagradable que se expresa por tensión emocional y se acompaña por manifestaciones somáticas; es una emoción normal cuya función es activadora y facilita la función de respuesta del individuo, pero se considera patológica cuando es excesiva en frecuencia o 
duración, o se asocia con estímulos que no representan una amenaza real produciendo alteraciones en el funcionamiento emocional de la persona (Agudelo, Buela-Casal, \& Donald, 2007).

Los padecimientos asociados con trastornos de ansiedad representan un desafío para los profesionales de la Psicología. Existen datos que indican que en México la prevalencia de trastornos de ansiedad generalizada ocurre entre el $20 \%$ de la población adulta, de estos casos, la prevalencia es más frecuente en las mujeres (Jiménez, 2014).

Para establecer con claridad la contribución de los factores de riesgo familiares para el desarrollo de la agresividad infantil, necesariamente se tiene que evaluar la contribución que los problemas emocionales comparten con otro factor de riesgo crucial en el ambiente emocional negativo que ocurre en el entorno familiar: la violencia marital. La violencia marital es un factor de riesgo para los problemas conductuales y emocionales de los niños, se caracteriza por tensión y peleas físicas entre los padres, desacuerdos para tomar decisiones en los asuntos de la vida familiar. Este es un problema prioritario de salud pública a nivel mundial, de acuerdo con algunos datos en América Latina, una de cada tres mujeres ha sido víctima de violencia la cual proviene en la mayoría de los casos del cónyuge o de algún familiar cercano (Mora, Natera, Tiburcio, \& Juárez, 2008).

En México existen datos que indican que el $43 \%$ de las mujeres de más de quince años sufre de violencia de pareja. En cuanto a los diferentes tipos de violencia, $32 \%$ es de tipo violencia emocional; $23 \%$, violencia económica; $6 \%$, violencia sexual y $9 \%$, violencia física (Cacique, 2017). La violencia marital tiene consecuencias sobre los problemas de conducta externalizada en los niños (Bradford et al., 2003), y se asocia estrechamente con otro factor de riesgo importante para la psicopatología infantil: las prácticas de disciplina severa.
El concepto prácticas parentales es tema central para los teóricos del desarrollo que analizan el impacto que tienen en el desarrollo psicosocial de los hijos (Andrade, Betancourt, Vallejo, Segura, \& Rojas, 2012). En este contexto, las prácticas de disciplina severa son la amplia gama de comportamientos parentales agresivos que tienen como propósito controlar la conducta de los hijos. Sus consecuencias son altamente dañinas tanto para los hijos como para los mismos padres, no obstante, el uso de estas prácticas es habitual a lo largo del mundo (Firmin \& Castle, 2008; Jiménez \& Flores, 2014; SaucedaGarcía, Olivo-Gutiérrez, Gutiérrez, \& MaldonadoDurán, 2007; So-Kum, 2006). Dada su alta frecuencia, la agresión que los padres ejercen para disciplinar a sus hijos se ha considerado común a casi todas las sociedades humanas (Cabrera-García, 2007; Corina, 2010).

Para algunos padres, las prácticas de disciplina severa son estrategias indeseables, mientras que para otros pueden llegar a ser estrategias eficaces para hacerse obedecer por sus hijos y están de acuerdo en que el trato severo es elemento indispensable en su desempeño como padres. Firmin y Castle (2008) encontraron que una alta proporción de padres creen que los golpes y azotes rigurosos no causan daño a los niños y que para ejercer la paternidad deben ser rudos y severos. Puede suponerse que la disciplina severa se fundamenta en la creencia de que es la mejor manera de controlar a los hijos (Cova et al., 2019).

En este cuerpo teórico, el control parental es un concepto relevante (Andrade et al., 2012) que se manifiesta como la disciplina rígida, la coerción, la inducción de la culpa, el retiro del afecto, el control hostil y el castigo (Betancourt \& Andrade, 2011). El castigo es el punto central en la interacción padreshijos. Entre las formas de castigo que causan mayor daño a los hijos, Buss (1961) clasificó a la agresión física, que se caracteriza por causar dolor y daño hacia el hijo, y la agresión verbal, en la que predomina el repudio y la amenaza; la hostilidad juega un papel central en estas formas de castigo. Prevalecen la 
presión o manipulación emocional y psicológica de los padres hacia sus hijos, y pocas expresiones de independencia y fomento de la autonomía (Andrade et al., 2012; Guevara, 2007).

En el presente estudio se considera que las madres son quienes aplican con mayor frecuencia las estrategias de disciplina y el castigo físico debido a que ellas tienen a su cargo la mayor parte de las labores de la crianza y el cuidado de los hijos. En la población mexicana las madres de los niños son el cuidador primario, quienes ante las conductas inadecuadas de sus hijos deciden cómo disciplinarlos (Sauceda-García et al., 2007).

El problema con que se enfrentan los investigadores es explicar en qué medida cada uno de los factores de riesgo identificados contribuye en la manifestación de los problemas infantiles. Con este propósito se han desarrollado modelos explicativos que permiten analizar el impacto de los factores de riesgo familiares sobre los problemas de conducta infantil, combinando variables para diseñar modelos y someterlos al escrutinio empírico.

El trabajo desarrollado por Ginsburg et al. (2004) tuvo como propósito analizar conductas parentales y trastornos por ansiedad en las madres. En dicho estudio, encontraron mayor probabilidad para que las madres ansiosas desarrollen síntomas externalizados en sus hijos.

El modelo coercitivo (Reid \& Patterson, 1989) explica la conducta agresiva durante la infancia y la adolescencia evaluando los efectos de las prácticas parentales sobre la conducta agresiva infantil. Este modelo supone que variables como la conducta antisocial de los padres y los conflictos entre los padres influyen sistemáticamente en el desarrollo de la agresión infantil, esta influencia es mediada por prácticas deficientes de disciplina. Las prácticas de disciplina severa son variables proximales que ocurren durante el desarrollo temprano y son fuertes predictores de la conducta agresiva. En el modelo coercitivo la conducta agresiva se aprende, fortalece y continúa mediante la interacción social en la que se ha establecido. Cuando las interacciones que ocurren entre los padres y sus hijos son agresivas e irritables se activa el ciclo coercitivo. Durante estas interacciones es decisiva la importancia que los padres demuestren al comportamiento agresivo e irritable de los niños, el niño aprende el comportamiento agresivo como una manera de relacionarse con los demás. Por otro lado, el modelo estructural de agresividad infantil de Chang et al. (2003) considera variables del sistema familiar controlado por conflicto marital y depresión parental y con efectos directos e indirectos sobre la conducta agresiva infantil. Existen datos que indican que la disciplina severa es una variable mediadora entre las deficiencias parentales y la agresividad infantil (Jiménez, 2014).

De la misma manera, se han diseñado modelos conceptuales que articulan las evidencias empíricas en un modelo general que permite la explicación sistematizada a partir de cada una de las interacciones analizadas. Ante esto, el modelo ecológico de desarrollo humano es un cuerpo de conocimientos centrados en el problema de la interacción que los sujetos tienen con escenarios conductuales (Mercado, Landázuri, \& Terán, 2001). En este modelo se analiza la interacción social, el intercambio de comportamientos verbales, gestuales o motores; los papeles que desempeñan tanto el emisor como el receptor, llegando a establecerse una forma de control mutuo, de manera que los actos de un individuo contribuyen a la dirección y control de los actos del otro. La orientación ecológica asume que lo que cuenta para la conducta y el desarrollo es el ambiente como se percibe, más que cómo pueda existir en la realidad objetiva (Sánchez, 2018).

La investigación aplicada a niños de edad escolar es una tarea que exige la construcción de un esquema teórico que permita el análisis sistemático de estos contextos, sus interconexiones y los procesos a través de los cuales estas estructuras y vinculaciones afectan el curso del desarrollo. 
Con base en lo expuesto hasta esta parte, pueden concretarse las siguientes hipótesis. Primero, los trastornos de ansiedad manifestados por las madres y los padres afectan negativamente el desarrollo conductual de los niños. Las madres ansiosas tienen mayor probabilidad de ocasionar conductas externalizadas en sus hijos. Segundo, la violencia marital es un constructo válido y universal que tiene efectos sobre problemas de conducta y emocionales en los niños y los adolescentes. Tercero, las prácticas de disciplina severa son variables mediadoras entre la violencia marital y los problemas de conducta y emocionales de los niños y los adolescentes. Finalmente, las prácticas de disciplina severa dan lugar a conducta agresiva en los niños.

Los supuestos teóricos y los modelos que explican la psicopatología infantil están de acuerdo en el vínculo que ocurre con los factores de riesgo familiares. Sin embargo, hay controversia acerca de la naturaleza de las trayectorias de las variables. Concretamente, cómo se comportan las trayectorias directas e indirectas de las variables en el modelo.

Los factores de riesgo familiares cuentan con amplio respaldo empírico. Existen datos que vinculan la conducta agresiva de los niños con los comportamientos violentos que ocurren entre los padres (Jiménez, 2014; Sánchez, 2018), los síntomas de ansiedad manifestados principalmente por las madres de estos niños (Ginsburg et al., 2004; Krahé, Bondü, Höse, \& Esser, 2014) y la severidad durante el ejercicio de las prácticas de disciplina (Chang et al., 2003; Forehand, Lafko, Parent, \& Burt, 2014).

No obstante, los hallazgos no son claros, particularmente al intentar explicar las trayectorias que estos constructos guardan entre sí. Con frecuencia se ha llegado a plantear que estas disfunciones parentales afectan positivamente el desarrollo de conductas de agresión en los niños (Fagan \& Benedini, 2016), pero aún no se ha establecido claramente cómo son las trayectorias entre estas variables. Mientras que algunos estudios proponen efectos directos entre las variables, otros han logrado identificar que las prácticas de disciplina parental son mediadoras entre variables parentales distales y los problemas de conducta infantil (Jiménez, 2014). Por lo cual, es conveniente distinguir las trayectorias y los efectos que ocurren durante la interacción entre estas variables. Por lo anterior, el presente estudio se apoya en las evidencias que han demostrado que los factores de riesgo familiares influyen en la manifestación de la conducta agresiva infantil. Para ello, se utiliza el planteamiento desarrollado por Reid y Patterson (1989) en su modelo ambiental de desarrollo psicosocial, donde la disciplina severa es una variable proximal de interacción social, mientras que los síntomas de ansiedad en las madres y la violencia marital son determinantes distales que activan un ciclo coercitivo que continua en la interacción social en la que se ha establecido dando lugar a comportamientos agresivos en los niños.

Nuestra pregunta de investigación se expresa de la siguiente manera: ¿En qué medida se presenta una adecuación en términos estadísticos y conceptuales del modelo propuesto acerca de la conducta agresiva infantil tomando en cuenta variables de ansiedad en las madres, violencia marital y prácticas de disciplina severa? De acuerdo a lo anterior, el objetivo general del presente estudio es construir un modelo explicativo de la agresividad infantil y su relación con factores de riesgo familiares, esto mediante una evaluación cuantitativa del modelo elaborado y la identificación de su adecuación en términos de análisis estadísticos de ajuste y su congruencia teórica.

El marco de hipótesis que orienta las relaciones exploradas en el modelo explicativo, son las siguientes:

Hipótesis 1: la ansiedad de las madres y la violencia marital tendrán efectos indirectos y positivos sobre la conducta agresiva infantil, mediada por las prácticas de disciplina severa.

Hipótesis 2: se espera encontrar una relación positiva entre los síntomas de ansiedad materna y la 
violencia marital; esto significa que a medida que aumentan los síntomas de ansiedad en las madres, mayor será la medida de la violencia marital.

Hipótesis 3: la sintomatología ansiosa de las madres tendrá efectos directos y positivos sobre la disciplina severa; esto implica que a medida que aumenta la ansiedad de las madres, mayor será la severidad en las prácticas disciplinarias hacia los hijos.

Hipótesis 4: la violencia marital tendrá efectos positivos sobre la disciplina severa; esto significa que a medida que aumenta la violencia marital, también aumenta la medida de la disciplina severa.

Para focalizar los constructos del modelo propuesto, y en concordancia con la revisión teórica efectuada, se eligieron las definiciones asociadas a las medidas elegidas en el presente estudio. Respecto a la variable criterio conducta agresiva (CAGRSIV), esta es un síndrome que incluye conductas de intimidación, agresión física, peleas, comportamientos violentos dirigidos hacia los demás, amenazas verbales, arrebatos explosivos de destrucción de propiedad y conducta de autodaño (Achenbach \& Rescorla, 2001).

Respecto a las variables predictoras, una de ellas es la ansiedad (específicamente, síntomas de ansiedad), la cual es una emoción caracterizada por sentimientos de tensión, pensamientos de preocupación y cambios físicos (Durand et al., 2007; Compas \& Gotlib, 2003). Estos síntomas se manifiestan a partir de cuatro indicadores (Robles, Varela, Jurado, \& Páez, 2001): nivel de pensamientos y preocupaciones (SUBJETIV), reacciones fisiológicas (NEUROFIS), reacciones no controlables reguladas por el sistema autónomo (AUTONOMI) y pensamientos de desesperación y angustia (PANICO). La siguiente variable predictora violencia marital hace referencia a la interacción entre los padres y expresa desacuerdos acerca de los asuntos en la vida familiar; además, es perjudicial, frecuente y crónica (Mora et al., 2008). Se clasifica en violencia emocional y psicológica (AGRESIO), conductas de coerción sexual (COERCI) y conductas de alto riesgo o violencia extrema entre la pareja (VIOLRIES). Finalmente, las prácticas de disciplina severa consisten en una gama de conductas caracterizadas por reacciones negativas conductuales y emocionales de los padres hacia sus hijos (Jiménez-Flores \& Flores-Herrera, 2014). Son estrategias que utilizan las madres para controlar la conducta de sus hijos a partir de cinco factores: (1) disciplina hostil (DISCHOST), que es una combinación de prácticas de disciplina física y verbal; (2) disciplina severa con agresión física (DISFISI), las cuales son conductas de castigo físico hacia el niño; (3) disciplina severa con agresión verbal (DAGVE), delimitada a conductas de castigo principalmente verbal y poco castigo físico; (4) castigo corporal moderado (CASTIGOC), que implica el uso de castigo físico pero en menor gradiente que los primeros factores; y (5) castigo verbal moderado (CASTIGOV), que es el uso de agresión verbal en menor gradiente.

\section{Método}

\section{Participantes}

La población del estudio estuvo compuesta por madres de familia del Estado de México, distribuidas en escuelas públicas primarias de diferentes colonias y de estatus socioeconómico predominante en el nivel. La selección de las participantes se realizó por muestreo aleatorio. La muestra de participantes seleccionada estuvo compuesta por 409 mujeres de clase trabajadora de poblaciones con bajos recursos económicos, seleccionadas con los siguientes criterios de inclusión: participación voluntaria, dedicación parcial o completa al hogar, y ser madres de niños entre 6 y 12 años. La edad promedio fue 34 años $(D E=6.5$, mínimo $=28$, máximo $=40)$. Todas ellas madres de alumnos que estudiaban en escuelas primarias públicas en el Estado de México. Respecto a los estudios logrados, el 25\% fue de nivel primaria, el $43 \%$ de secundaria, el $14 \%$ de bachillerato, el 12\% de una carrera técnica, el 5\% profesional y el 1\% informaron no tener ningún tipo de estudio. En lo 
referente a su estado civil se encontró que el $48 \%$ eran casadas, el 43\% vivían en unión libre, 5\% vivían en segunda unión libre y 4\% mantenían otro tipo de relación de pareja.

\section{Instrumentos}

Listado de conductas infantiles (Children Behavior Check List, CBCL/6-18; Achenbach \& Rescorla, 2001). Es un instrumento que pregunta a los padres sobre los síntomas internalizados y externalizados del niño. Usa una escala de respuesta desde 0 («no es verdad») hasta 2 («muy verdadero o frecuentemente es verdad»). Para este estudio se utilizó la subescala de conducta agresiva, la cual se configura por 18 reactivos que arrojan una calificación total posible de 36 puntos ( $\alpha=.91$, validez de criterio). Una calificación de 12 puntos o menos coloca al niño en área de normalidad. En el presente estudio, la consistencia interna fue $\alpha=.90$ (IC 95\%: .88, .91).

Inventario de ansiedad de Beck (BAI; Beck, Epstein, Brown, \& Steer, 1988). Cuestionario de autoinforme que mide los síntomas de ansiedad. Consta de 21 reactivos, cada uno de los cuales se califica en una escala de 4 puntos, desde 0 («poco o nada») hasta 3 («severamente») el síntoma en cuestión. El puntaje total tiene adecuada consistencia interna ( $\alpha=.83$ ) y confiabilidad test retest aceptable (correlación intraclase $r=.75$ ). Se utilizó la adaptación mexicana de Robles et al. (2001). En el presente estudio, la consistencia interna fue $\alpha=.93$ (IC 95\%: $.91, .93)$.

Escala de tácticas de conflicto (Conflict Tactics Scale; Mora et al., 2008). Instrumento utilizado para obtener información sobre la violencia masculina dirigida hacia la mujer. Se compone de tres dimensiones: (1) violencia emocional y física, (2) acoso sexual y (3) violencia de alto riesgo. Está formado por 21 reactivos en escala ordinal que exploran las tácticas ejercidas por la pareja masculina hacia las mujeres. El escalamiento ordinal de respuesta va desde 0 («nunca») hasta 2 («en últimos 12 meses»).
Mora et al. (2008) reporta adecuada estructura y consistencia interna global $(\alpha=.93)$. En el presente estudio, la consistencia interna fue $\alpha=.96$ (IC 95\%: $.95, .96)$.

Lista de prácticas de disciplina severa (JiménezFlores \& Flores-Herrera, 2014). Instrumento utilizado para valorar las prácticas disciplinarias que ejercen los padres sobre los hijos. Se forma por 19 reactivos en formato de escala Likert con tres opciones de respuesta. El análisis factorial arrojó cinco factores: disciplina hostil, disciplina severa con agresión física, disciplina severa principalmente con agresión verbal, castigo corporal moderado y castigo verbal moderado; cuenta con consistencia interna total de $\alpha=.88$. En el presente estudio, la consistencia interna fue $\alpha=$ .87 (IC 95\%: .85, .88).

\section{Procedimiento}

\section{Diseño de la investigación}

Fue un estudio de una cohorte de tipo transversal, prospectivo y explicativo (Bazán, Sánchez, \& Castañeda, 2007), que buscaba establecer relaciones de causalidad probabilística entre distintos factores asociados con la conducta agresiva infantil.

\section{Recolección de datos}

Para recolectar los datos se capacitó a un grupo estudiantes de Psicología. Previamente se gestionaron los espacios correspondientes con las autoridades escolares. Los instrumentos se aplicaron de manera grupal, a las participantes que no entendían las instrucciones se les apoyó de manera individual. Respecto a las consideraciones éticas, se explicó que la participación era voluntaria y si no querían participar, podían retirarse; quienes decidieron continuar firmaron un consentimiento informado.

\section{Análisis de datos}

Se aplicó el modelamiento de ecuaciones estructurales mediante el programa EQS 6.2 (Bentler 
\& Wu, 2002). Se utilizó el estimador de máxima verosimilitud robusto, y el ajuste se evaluó con la prueba $\chi^{2}$ y coeficientes aproximados de ajuste práctico (CFI, NNFI y RMSEA). Para la interpretación de los resultados, se obtuvieron correlaciones latentes y coeficientes beta.

\section{Resultados}

En primer término, el objetivo fue evaluar el comportamiento de cada variable, con este propósito se realizó el análisis descriptivo para cada una de las variables incluidas en el modelo.

\section{Análisis descriptivo}

\section{Conducta agresiva}

En el CBCL se considera rango clínico a partir de una puntuación de 12, los datos arrojaron que el 19\% de esta muestra (77 niños) se ubica en tal rango clínico.

\section{Ansiedad materna}

De acuerdo con los datos normativos para población mexicana, las frecuencias se agrupan de acuerdo a lo expuesto en la Tabla 1 . Puede observarse que $22.7 \%$ de estas mujeres manifiestan síntomas de ansiedad en nivel elevado, este porcentaje de población merece de atención especializada.

\section{Tabla 1}

Ansiedad: distribución de acuerdo con los datos normativos

\begin{tabular}{lcc}
\hline \multicolumn{1}{c}{ Clasificación } & $n$ & $\%$ \\
\hline Ansiedad mínima & 173 & 42.3 \\
Ansiedad leve & 143 & 35 \\
Ansiedad moderada & 68 & 16.6 \\
Ansiedad severa & 25 & 6.1 \\
\hline
\end{tabular}

\section{Violencia marital}

Los datos indican que una parte de esta muestra sufre de conductas tipificadas como violencia marital (Tabla 2). En 45 casos (rango percentil 94) de esta muestra ocurren problemas de violencia marital. Algunas de estas participantes sufren de los tres tipos de violencia marital.

\section{Prácticas de disciplina severa}

En la distribución de frecuencias (Tabla 2) se puede observar que 51 de estas madres (rango percentil 90) aplican con bastante severidad sus prácticas disciplinarias. Un análisis más detallado de los factores encontrados en esta variable indica que (Tabla 2), por ejemplo, en el factor de disciplina hostil, 55 madres (ubicadas sobre el percentil 94) practican medidas disciplinarias de rechazo verbal y castigo físico con alta severidad. También se observa que 32 madres se ubican en un nivel elevado de severidad en los comportamientos clasificados en el factor disciplina severa con agresión física. De la misma manera se puede observar que en una frecuencia menor, 20 madres se ubican con bastante severidad en el factor disciplina severa principalmente con agresión verbal. Asimismo, 27 madres practican con severidad el castigo corporal moderado (percentil 98) y 22 madres practican con severidad el castigo verbal moderado (percentil 98).

\section{Modelamiento de la agresividad}

El objetivo general fue someter a prueba un modelo de relaciones estructurales entre variables latentes: ansiedad materna, violencia marital, disciplina severa y conducta agresiva infantil. En la especificación del modelo propuesto, los síntomas de ansiedad materna y la violencia marital son variables exógenas que tienen efectos indirectos sobre la conducta agresiva infantil que es la variable dependiente. Estos efectos son mediados por las prácticas de disciplina severa (Figura 1). 
Tabla 2

Resultados descripticos para violencia marital y prácticas de disciplina severa

\begin{tabular}{lcccc}
\multicolumn{1}{c}{ Factor } & $M$ & $D E$ & Percentil & $n(\%)$ \\
\hline Violencia marital & & & & \\
$\quad$ Violencia emocional y física & 2.85 & 3.99 & 94 & $31(\mathrm{x} \%)$ \\
Coerción sexual & .44 & 1.6 & 95 & $23(\mathrm{x} \%)$ \\
Violencia de alto riesgo & .54 & 1.96 & 95 & \\
& & & & $55(\mathrm{x} \%)$ \\
Prácticas de disciplina severa & & & & $32(\mathrm{x} \%)$ \\
Disciplina hostil & 3.33 & 1.82 & 94 & $20(\mathrm{x} \%)$ \\
Disciplina severa física & 1.22 & .957 & 97 & $27(\mathrm{x} \%)$ \\
Disciplina con agresión verbal & 1.19 & 1.30 & 98 & $22(\mathrm{x} \%)$ \\
Castigo corporal moderado & .81 & .97 & 98 & 98 \\
Castigo verbal moderado & .66 & .94 & & \\
\hline
\end{tabular}

Nota: $M=$ Media; $D E=$ Desviación estándar.

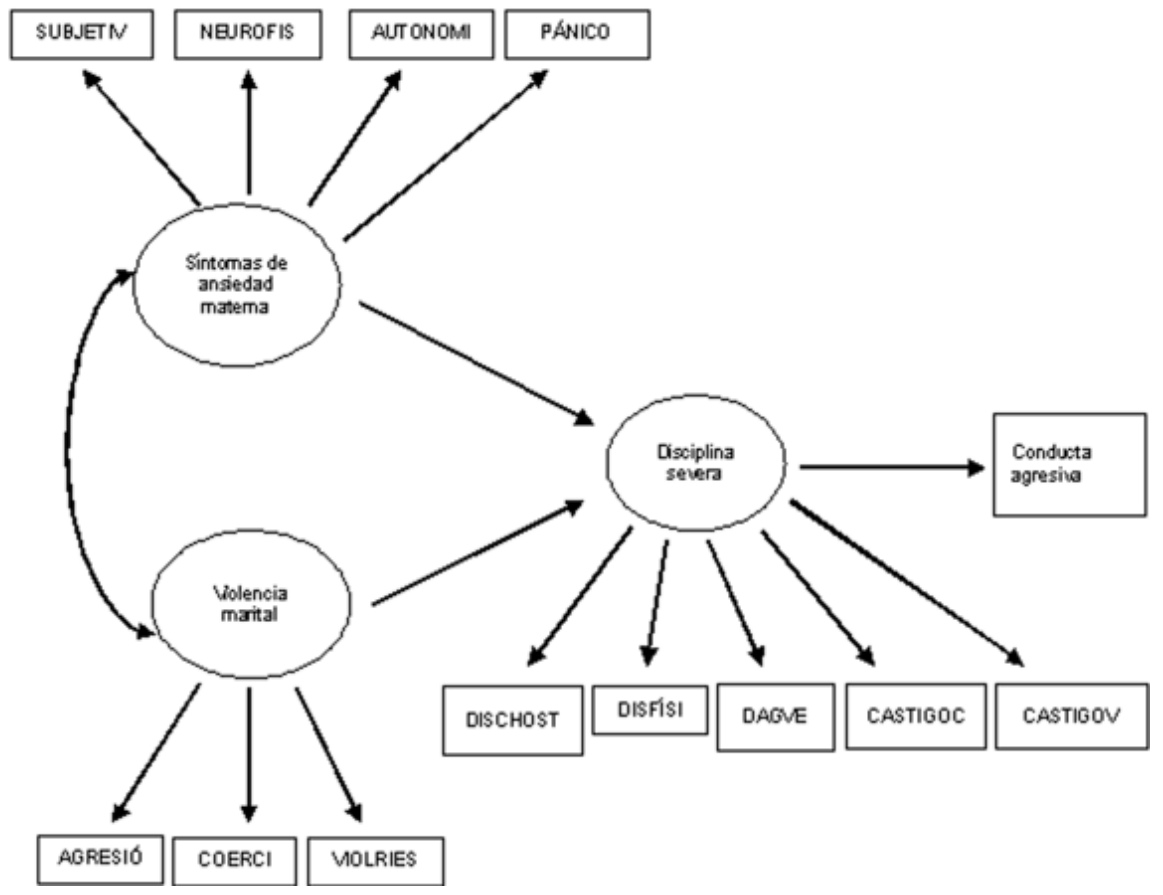

Figura 1. Modelo hipotético estructural de factores de riesgo familiares asociados a la conducta agresiva infantil 
El modelo muestra una adecuada bondad de ajuste $\left(\chi^{2}=207.14, p<.05, g l=62\right)$, con indicadores prácticos de bondad de ajuste también adecuados $(\mathrm{CFI}=.95$, NNFI $=.94$, RMSEA $=.08)$. El modelo obtenido es similar al modelo propuesto de relaciones estructurales entre las variables incluidas en este estudio (Kline, 1998). De tal forma, la bondad de ajuste del modelo obtenido respecto del modelo hipotético que se probó, mostró una coherencia teóricamente plausible.

En cuanto a la relación estructural entre los constructos incluidos (Figura 2), el modelo muestra que existe una relación significativa entre las variables exógenas, ansiedad materna y violencia marital ( $r=$ .23); tanto la ansiedad materna como la violencia marital influyen sobre las prácticas de disciplina severa ( $\beta=.44$ y $\beta=.10$, respectivamente). Las prácticas de disciplina severa influyen sobre la conducta agresiva de los niños ( $\beta=.59$ ). La disciplina severa es variable mediadora entre la ansiedad materna, la violencia marital y la conducta agresiva infantil. La variabilidad de la conducta agresiva es explicada en $34 \%$ por el modelo ecológico de factores de riesgo familiares (Figura 2). De acuerdo con el modelo resultante puede distinguirse que las cuatro variables en el modelo hipotético lograron configurarse significativamente. Asimismo, se puede observar que la variable prácticas parentales es la que mejor explica a la conducta agresiva infantil es la disciplina severa, que se refiere a las estrategias que las madres utilizan para controlar la conducta de los hijos. Se observa que la variable disciplina severa es influida principalmente por la variable ansiedad materna, seguida por la variable violencia marital. Todas las relaciones establecidas son significativas.

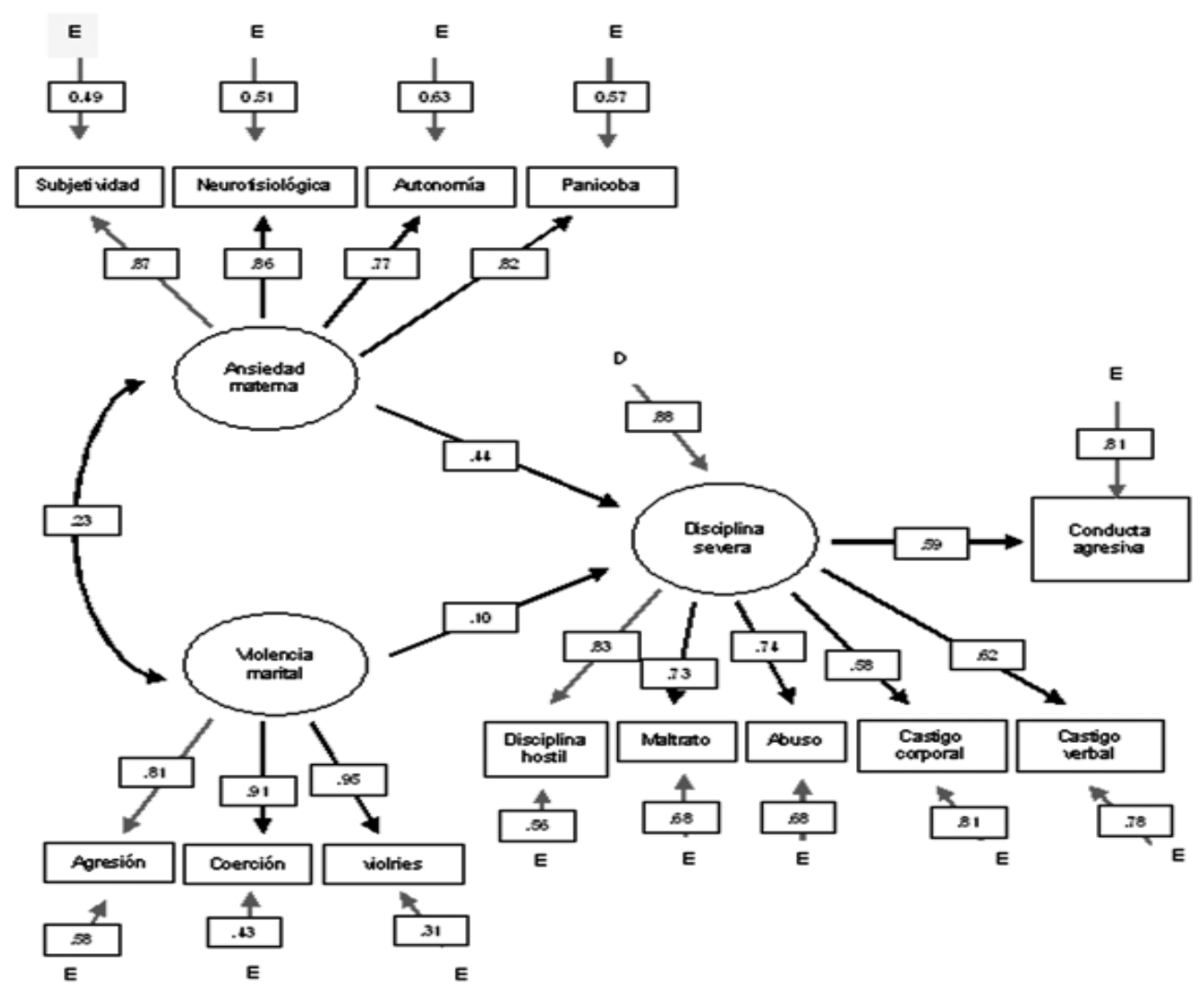

Figura 2. Modelo estructural de conducta agresiva $\left(x^{2}=207.14, p<.00\right.$, g.l = 62; CFI = .95; NNFI = .94; RMSEA $=.08)$. 


\section{Discusión}

El objetivo de este trabajo fue construir un modelo ecológico explicativo de la agresividad infantil con la finalidad de que los hallazgos encontrados aporten evidencias teóricas y metodológicas para la toma de decisiones en la solución de problemas ocasionados por las conductas agresivas de los niños y la interacción que guardan con su ambiente familiar. También se hicieron análisis descriptivos para conocer el estatus de los participantes respecto a las variables evaluadas. Los resultados descriptivos y del modelamiento se explican a continuación.

\section{Sobre los resultados descriptivos}

Respecto a la conducta de agresión infantil, los datos encontrados indican que la quinta parte de la población son niños que requieren urgentemente de atención especializada. Estos resultados se relacionan con hallazgos referidos en estudios previos tanto a nivel nacional (Jiménez, 2014; Sánchez, 2018) como global (Cabrera-García, 2007; Chang et al., 2003). En relación a las prácticas de disciplina severa, se encontraron comportamientos de desprecio hacia el hijo y expresiones matizadas de ira, es importante resaltar que predomina la hostilidad verbal. Los datos dan cuenta de un porcentaje de madres que aceptan llevar a cabo prácticas de disciplina severa. No obstante, debe reconocerse que más de la mitad de las participantes negaron ubicarse en alguna de estas dimensiones. Cabe la posibilidad de que los datos sean más altos en la severidad de la variable. Efectivamente, los resultados de distintas investigaciones son consistentes en cuanto a que el castigo físico y el castigo verbal se asocian con conductas agresivas en los niños (Flores et al., 2017). En el caso del presente estudio, la variable de prácticas de disciplina severa fue la que se asoció con mayor firmeza a la conducta agresiva infantil.

Por otro lado, sobre los síntomas de ansiedad en las madres, los datos obtenidos apoyan las evidencias de estudios previos cuyo propósito ha sido medir indicadores de ansiedad en mujeres (Ginsburg et al., 2004) y están en línea con el supuesto de que entre los trastornos emocionales en las mujeres, los síntomas de ansiedad son los que se observan con mayor frecuencia. Cuando las mujeres manifiestan síntomas de ansiedad desde moderada a grave, tienden a ser menos cálidas con sus hijos, no fomentan su autonomía y los someten a situaciones estresantes. Algunos estudios (Reppeti et al., 2002) dan cuenta que las madres con este tipo de trastornos con frecuencia desarrollan trastornos externalizados en los niños. En último lugar, sobre la descripción de la violencia marital, cabe mencionar, con base en los resultados obtenidos, que hubo dimensiones completas de la violencia marital cuya incidencia fue muy baja, como en el caso de la coerción sexual y la violencia de alto riesgo. No obstante, si los porcentajes observados se mantienen en la población general, la frecuencia de estos casos es preocupante: $5 \%$ informó haber padecido de violencia marital de alto riesgo, 7.7\% aceptaron haber padecido de coerción sexual y $10 \%$ de violencia emocional y física. Es pertinente mencionar que la violencia que ocurre en la relación de pareja tiende a ser ocultada, principalmente por las mujeres, claramente los hombres no la denuncian. Otra explicación de la baja frecuencia de casos informados consiste en la aceptación cultural de las mujeres hacia ciertas conductas agresivas por parte del cónyuge (Mora et al., 2008). Lo relevante de los resultados obtenidos es que una de cada diez mujeres encuestadas reconoció haber padecido de violencia emocional y física; y una de cada veinte de estas mujeres aceptó agresión de alto riesgo por parte de su pareja. Si estos datos se mantienen en población general, el porcentaje de mujeres afectadas representa un gran reto para los especialistas del área.

\section{Sobre el modelamiento}

A continuación, se da respuesta a las hipótesis de investigación, a la vez que se discuten los resultados a la luz de los planteamientos teóricos que les dieron sustento. 
Hipótesis 1. La ansiedad de las madres y la violencia marital tienen efectos indirectos sobre la conducta agresiva infantil mediada por las prácticas de disciplina severa. Este planteamiento se basa en los estudios desarrollados por Chang et al. (2003). Para analizar este supuesto es pertinente observar la predicción de la conducta agresiva a partir de las prácticas de disciplina severa $(\beta=.59)$. Ya en el modelo estructural, los síntomas de ansiedad materna ( $\beta=.44)$ predicen a las prácticas de disciplina severa, mientras que la violencia marital $(\beta=.10)$ también es un predictor de disciplina severa. En este orden, las prácticas de disciplina severa son el constructo que mejor predice a la conducta agresiva, mientras que los efectos de las variables exógenas son mediados por la variable endógena.

Hipótesis 2. Los resultados confirman que ocurre una asociación positiva y significativa entre los síntomas de ansiedad y la violencia marital, aunque debe observarse que la magnitud de la relación es baja ( $r=$ .23), por lo tanto la variabilidad explicada es 5.29\%. Tanto en la sintomatología de ansiedad como en la violencia marital, los datos indican que las participantes consideradas en riesgo son una proporción baja comparada con el total; en la primera variable la proporción en riesgo fue de $22.7 \%$, mientras que en la segunda fue el $10 \%$. Es probable que estos datos hayan llevado a una correlación tan débil. Además, no todas las mujeres ansiosas se relacionan con episodios de violencia marital, aunque cabe la posibilidad que el $6.1 \%$ de mujeres que informaron padecer de síntomas graves de ansiedad pueda también incluirse en la clasificación de violencia marital extrema. Por otra parte, debe considerarse que en el modelo ecológico, estas variables son variables distales, condiciones que ya están dadas y que ocurren independientemente de los niños, aunque sí los afectan.

Hipótesis 3. Los síntomas de ansiedad materna influyen sobre las prácticas de disciplina severa. El planteamiento se formuló de acuerdo con los estudios de Chang et al. (2003) y Ginsburg et al. (2004), en los cuales se supone que las formas de controlar los comportamientos inadecuados de los niños están influidas por el estado de ansiedad manifestado por las madres. En los resultados se confirmó este planteamiento $(\beta=.44)$, y se encontró que cuando las madres padecen de síntomas de ansiedad tienden a ser más controladoras y a castigar con mayor severidad a sus hijos para controlar su comportamiento. El peso de la regresión es moderado, por lo que es importante considerar la influencia que tiene la sintomatología de ansiedad materna en la predicción de las prácticas de disciplina severa. En lo que corresponde al modelo de conducta agresiva, configuran una interacción importante para explicar la dependencia entre variables exógenas y variables endógenas. Estos datos están de acuerdo con los hallazgos referidos en la literatura especializada.

Hipótesis 4. El planteamiento consistió en que la violencia que ocurre entre los padres afecta las prácticas de disciplina severa, esto supone que los desacuerdos y conflictos que ocurren entre la pareja llevan a que se ejerzan prácticas disciplinarias agresivas hacia los hijos (Cabrera-García, 2007). Pudo comprobarse que la violencia marital es un predictor de disciplina severa, aunque la dependencia observada fue baja $(\beta=.10)$. Un factor que probablemente influyó para obtener este coeficiente puede ser la baja incidencia de casos clasificados en la variable violencia marital.

De acuerdo con los resultados, se ha obtenido lo siguiente. a) La evaluación del modelo explicativo de la agresividad infantil indica que las prácticas de disciplina severa son comportamientos de relevancia para el modelo ecológico de agresión infantil. En el contexto del modelo ecológico de desarrollo se perfila a las variables proximales con mayor nivel de influencia para la conducta agresiva de los niños, en comparación con las variables distales. b) La sintomatología ansiosa de las madres influye positivamente sobre la conducta agresiva infantil con la mediación de las prácticas de disciplina severa. Los problemas de ansiedad de las madres son predictores potenciales de conducta agresiva en los niños 
(Ginsburg et al., 2004). Esto se debe principalmente a que estas mujeres manifiestan bajo nivel de afecto hacia sus hijos y porque no permiten la autonomía a sus hijos (Guevara, 2007). Los hallazgos del presente estudio concuerdan con estudios anteriores que informan que la sintomatología de ansiedad materna predice comportamientos agresivos en los niños (Langrock et al., 2002; Reppeti et al., 2002). c) La violencia marital influye sobre la conducta agresiva de los niños con la mediación de las prácticas de disciplina severa. Los hallazgos apoyan estudios que han documentado la fuerte asociación entre estas variables (Bradford et al., 2003).

Cabe resaltar que los planteamientos teóricos y las variables estudiadas se encuentran en un continuo teórico-metodológico enmarcado en el contexto del modelo ecológico denominado Modelo de interacción social para el desarrollo de conducta antisocial (Reid \& Patterson, 1989), en el cual las variables violencia marital y sintomatología de ansiedad materna son consideradas variables distales, mientras que las prácticas de disciplina severa son conceptuadas como variables proximales.

La posibilidad de su medición en un momento específico es gracias a que tanto las variables proximales como las distales pueden coincidir en un mismo momento y sus consecuencias pueden manifestarse en comportamientos concretos en los seres humanos, que en este caso es la conducta de agresión infantil. Los determinantes distales influyen en el desarrollo de comportamiento coercitivo solo hasta que los padres interactúen con sus hijos durante su vida diaria. Una conducta de interacción entre el padre y el hijo es precisamente el estilo disciplinario.

\section{Implicaciones del estudio}

A continuación, se presentan las aportaciones de la presente investigación de acuerdo con los datos obtenidos.

\section{Medición de la conducta agresiva infantil}

La medición de la conducta de agresión infantil es una aportación del presente trabajo. De acuerdo con los datos, la proporción de niños que manifiestan problemas de conducta agresiva clasificada como rango clínico es la quinta parte de la población infantil. Estos datos pueden utilizarse para diseñar propuestas de intervención.

\section{Evaluación de la sintomatología de ansiedad}

La evaluación del nivel de ansiedad informado en las mujeres en la zona donde se levantaron los datos es escaza la valoración para los niveles de ansiedad en la población, formada principalmente por amas de casa y cuidadoras primarias de sus hijos. Por esta razón, los datos encontrados son útiles para diseñar intervenciones, incluso a nivel comunitario.

\section{Evaluación de la violencia marital}

Los datos referentes a esta variable son bastante extremos. Indican una alta proporción que refiere no manifestar conductas de violencia marital. Es claro que hay que diseñar intervenciones para ese $10 \%$ de población afectada, y por toda la población que no respondió adecuadamente en esa parte del instrumento.

\section{Modelo ecológico de factores de riesgo familiares y conducta agresiva infantil}

En el modelo ecológico desarrollado por Jiménez (2014), se menciona que la interacción entre las variables proximales y las distales activan un ciclo coercitivo de conducta agresiva. De acuerdo con los datos, la variable proximal tuvo mayor influencia sobre la conducta agresiva. Es más importante la interacción que ocurre entre las madres y sus hijos cuando las primeras intentan controlarlos: las variables distales influyen, pero en menor medida.

De acuerdo con lo anterior, el modelo ecológico de conducta agresiva infantil está determinado por las 
interacciones que ocurren en el ambiente familiar, siendo la interacción durante las prácticas disciplinarias el elemento principal para mantener la agresión infantil. Los datos apoyan el supuesto de que la conducta agresiva infantil se aprende desde la interacción familiar durante la infancia (Compas \& Gotlib, 2003), etapa en que los padres, principalmente la madre, modelan y recompensan estas conductas.

En segundo término, se mencionan detalles referentes al modelo estadístico. A lo largo de la literatura se ha insistido en que correlación no significa determinación y que los factores distales no deben ser considerados como condiciones determinantes, sino como condiciones que los investigadores tienen que comprender y explicar (Sander \& McCarty, 2005). En este sentido, se han encontrado estudios que refieren distintas posturas respecto a las interacciones que ocurren entre las deficiencias parentales y la conducta de agresión infantil. En el modelo de Langrock et al. (2002), las variables parentales no tienen efectos directos sobre los problemas emocionales de los niños, los estilos de afrontamiento de los niños son variables mediadoras entre los comportamientos parentales y la conducta de los hijos. Por su parte, el modelo de Bradford et al. (2003) indica que ocurren efectos directos e indirectos entre las deficiencias parentales (violencia marital) y la psicopatología infantil. En este sentido, las prácticas de disciplina tienen efectos mediadores entre la violencia marital y los problemas de conducta infantil.

Finalmente, la confirmación de los resultados de la influencia de estas variables parentales sobre el desarrollo de problemas de conducta requiere de nuevas aplicaciones sistematizadas y el desarrollo de instrumentos de medición con mejores propiedades psicométricas. La relevancia de los síntomas de ansiedad de las madres y de la severidad ejercida durante las prácticas disciplinarias para predecir la conducta agresiva confirma hallazgos referidos en estudios previos.

\section{Limitaciones}

Pese a la relevancia de los hallazgos de este estudio, es conveniente señalar algunos aspectos a mejorar en el futuro. En primer lugar, los datos de la evaluación de la conducta de agresión infantil fueron obtenidos mediante la información proporcionada por las madres de estos niños. Ello implica que es probable que la frecuencia de niños agresivos sea mayor. Una posible fuente de error puede ser que las madres hayan ocultado información por temor a decisiones institucionales al interior de las escuelas. Otra fuente de error puede atribuirse al desconocimiento de las madres sobre las conductas de sus hijos. Otro punto consiste en un hecho característico de la población mexicana: la conducta agresiva es aceptada y fomentada. En segundo lugar, respecto al uso de autoinformes, su empleo implica un proceso de inferencia, especialmente cuando se trata de conductas típicas o en algunas situaciones indeseables. El autoinforme puede llevar un sesgo por parte del actor. Para subsanar este hecho, es recomendable que posteriormente, se lleve a efecto otro tipo de mediciones utilizando otros instrumentos, así como el uso de medidas directas creando situaciones pertinentes y bajo los principios de la ética. Finalmente, un punto que queda sometido a discusión es referido a los síntomas de ansiedad materna, aunque los datos son significativos en la relación que guardan con los problemas de conducta infantil, aún quedan preguntas por resolver. No todos los niños en alto riesgo desarrollan conducta agresiva, algunos niños poseen ciertas características que los hacen inmunes a los efectos dañinos de la ansiedad materna.

\section{Sugerencias}

Para futuros estudios se recomienda utilizar muestras provenientes de población clínica, particularmente porque hay mayor probabilidad de controlar las variables. Otra sugerencia para mejorar el estudio es el empleo de registros observacionales para medir las prácticas disciplinarias con el objeto de superar los sesgos derivados de las mediciones del 
autoinforme. En este sentido, se recomienda replicar este estudio con poblaciones amplias y comparar con datos aplicados con población clínica.

Asimismo, es recomendable replicar estudios analizando la conducta agresiva infantil con otros instrumentos, poblaciones amplias y de distintas zonas; además de utilizar recursos metodológicos de mayor precisión. Se considera conveniente continuar explorando la predicción de la disciplina severa en función de la violencia marital, asimismo, con el diseño de instrumentos de precisión y la réplica hacia otros tipos de población, seguramente se pueden lograr hallazgos que expliquen con mayor precisión estos fenómenos psicológicos.

Para futuros estudios, se recomienda utilizar muestras provenientes de población clínica, particularmente porque hay mayor probabilidad de controlar las variables. Otra sugerencia para mejorar el estudio es el empleo de registros observacionales para medir las prácticas disciplinarias, con el objeto de superar los sesgos derivados de las mediciones del autoinforme. Es recomendable aplicar estudios, incluyendo grupos amplios y de distintos estratos sociales, además, considerar los estilos disciplinarios de los padres.

De acuerdo con todo lo anterior, se ha expuesto un trabajo que aporta evidencias acerca de conductas que causan problemas y sufrimiento a los seres humanos. Las muestras elegidas son una pequeña parte de un universo de personas que demandan de la intervención efectiva para sus problemas de salud psicológica. Los datos obtenidos sirven, por un lado, para someter a prueba postulados científicos, y por otro, como indicadores que necesariamente tienen que derivar en beneficio de esos grupos focales, como los que se presentan en este trabajo, los cuales manifiestan discapacidades parentales, problemas emocionales y conductas de riesgo familiares. El seguimiento para el presente estudio está en función de identificar deficiencias y continuar profundizando en todos los aspectos mencionados con la firme convicción de que puedan traducirse en programas eficaces que solucionen los comportamientos disfuncionales de estas personas.

\section{Conflicto de intereses}

Los autores del presente estudio hacemos la aclaración que no existe conflicto de intereses de ningún tipo.

\section{Responsabilidad ética}

Para el manejo y la recolección de datos, los participantes firmaron una carta de consentimiento informado. En todo el proceso de la investigación fueron resguardados los datos de los participantes.

\section{Contribución de autoría}

JJF: concepción y diseño del estudio, recolección de los datos, interpretación de los datos y revisión final del manuscrito.

LMFH: revisión de análisis estadísticos y propiedades psicométricas de los instrumentos, revisión del manuscrito.

CMS: revisión del manuscrito y de los análisis estadísticos, índices de confiabilidad e intervalos de confianza.

\section{Referencias}

Achenbach, T. M., \& Rescorla, L. A. (2001). Manual for the Child Behavior Checklist. ASEBA: EE. UU.

Agudelo, D., Buela-Casal, G., \& Donald, S. (2007). Ansiedad y depresión: el problema de la diferenciación a través de sus síntomas. Salud Mental, 30(2), 33-41.

Andrade, P., Betancourt, D., Vallejo, A., Segura, B., \& Rojas, M. (2012). Prácticas parentales y sintomatología depresiva. Salud Mental, 35(1), 2936.

Bazán, A., Sánchez, B. A., \& Castañeda, S. (2007). Relación estructural entre apoyo familiar, nivel educativo de los padres, características del maestro 
y desempeño en lengua escrita. Revista Mexicana de Investigación Educativa, 12(33), 701-729. Recuperado de https://www.redalyc.org/ articulo.oa?id=14003312

Beck, A. T., Epstein, N., Brown, G., \& Steer, R. A. (1988). An Inventory for Measuring Clinical Anxiety: Psychometric Properties. Journal of Consulting and Clinical Psychology, 56(6), 893-897. doi: 10.1037/ /0022-006x.56.6.893

Bentler, P., \& Wu, E. (2002). EQS 6 for Windows. User's Guide. Encino, CA: Multivariate Software, Inc.

Benzies, K., Harrison, M., \& Magill-Evans, J. (2004). Parenting and Childhood Behavior Problems: Mother's and Father's Voices. Issues in Mental Health Nursing, 25(1), 9-24. doi: 10.1080/ 01612840490249028-21

Berkowitz, L. (2008). On the Consideration of Automatic as Well as Controlled Psychological Processes in Aggression. Aggressive Behavior, 34(2), 117-129. doi: 10.1002/ab.20244

Betancourt, D. (2007). Control parental y problemas internalizados $y$ externalizados en niños $y$ adolescentes (Tesis doctoral). Universidad Nacional Autónoma de México, México.

Betancourt, D., \& Andrade, P. (2011). Control parental y problemas emocionales y de conducta en adolescentes. Revista Colombiana de Psicología, 20(1), 27-47.

Bradford, K., Barber, B., Olsen, J., Maughan, S., Erickson, L., Ward, D., \& Stolz, H. (2003). A Multi-National Study of Interparental Conflict, Parenting, and Adolescent Functioning. Marriage \& Family Review, 35(3-4), 107-137. doi: 10.1300/j002v35n03_07

Buss, A. (1961). The Psychology of Aggression. New York: John Wiley \& Sons Inc. doi: 10.1037/11160000

Bustos-Aguayo, M., \& Flores-Herrera, M. L (2001). El papel del ambiente en el desarrollo y desempeño del niño. In L. M. Flores-Herrera \& J. M. Bustos-Aguayo (Comps.), Concepciones en la interacción social del niño (pp. 103-156). México: Facultad de Estudios Superiores Zaragoza, UNAM.
Cabrera-García, V. (2007). Relaciones maritales, relaciones parentales y su influencia en el ajuste psicológico de los hijos. In V. Cabrera-García \& P. Guevara (Eds.), Relaciones familiares y ajuste de psicológico: Dos estudios en adolescentes de familias colombianas (pp. 1-65). Bogotá: Universidad de los Andes.

Cacique, I. (2017). Fuentes y datos sobre la violencia contra las mujeres en México. Aprendizajes, dificultades y retos acumulados. Revista Internacional de Estadística y Geografía, 8(1), 616.

Chang, L., Dodge, K., Schwartz, D., \& McBride-Chang, C. (2003). Harsh Parenting in Relation to Child Emotion Regulation and Aggression. Journal of Family Psychology, 17(4), 598-606. doi: 10.1037/ 0893-3200.17.4.598

Compas, B. E., \& Gotlib, H. I. (2003). Introducción a la Psicología Clínica. México: McGraw Hill.

Corina, V. (2010). Escala de tolerancia parental hacia los comportamientos infantiles, elaboración y validación. Revista Latinoamericana de Psicología, 42(2), 203214.

Cova, F., Rincón, P., Bustos, C., Streiner, D., King, M., Saldivia, ... Novoa, C. (2019). Randomized Cluster Trial of a Parenting Program in Chile: Key Mediators in the Decrease in Behaviors Problems in Preschool Children. Clinical Child Psychology and Psychiatry, 1-13. https://doi.org/10.1177/1359104519864124

Durand, V. M., Barlow, H. D., \& Pecina-Hernández, J. (2007). Psicopatología: un enfoque integral de la Psicología anormal. México: Thomson.

Fagan, A. A., \& Benedini, K. M. (2016). How do FamilyFocused Prevention Programs Work? A Review of Mediating Mechanisms Associated with Reductions in youth Antisocial Behaviors. Clinical Child and Family Psychology Review, 19(4), 285-309. doi: 10.1007/s10567-016-0207-0

Fajardo, V., \& Hernández, L. (2008). Tratamiento cognitivo conductual de la conducta agresiva infantil. Revista Mexicana de Análisis de la Conducta, 34(2), 371-389. doi: 10.5514/rmac.v34.i2.16216 
Ferguson, K., Cassells, R., MacAllister, J., \& Evans, G. (2013). The Psysichal Environment and Child Development: An International Review. International Journal of Psychology, 48(4), 437-468. doi: 10.1080/00207594.2013.804190

Firmin, M. W., \& Castle, S. L. (2008). Early Childhood Discipline: A Review of the Literature. Journal of Research on Christian Education. 17(1), 107-129. doi: 10.1080/10656210801909715

Flores, L. M., Ruíz, M., Jiménez, J. \& Mercado, S. J. (2017). Influencia de las prácticas parentales y los problemas internalizados y externalizados en sus hijos adolescentes. In L. M. Flores \& J. Jiménez (Aut.), Funcionamiento familiar y escolar. Estudios evaluativos y de intervención (pp. 56-76). Facultad de Estudios Superiores Zaragoza, UNAM.

Flores, L. M., Villegas, P., Martínez, G., \& Palacios, P. (2017). Modelo psicoambiental de la agresión infantil. Revista Electrónica de Psicología de la FES Zaragoza, 7(14), 14-19. Recuperado de https:// www.zaragoza.unam.mx/wp-content/Portal2015/ publicaciones/revistas/rev_elec_psico/REP_14.pdf

Forehand, R., Lafko, N., Parent, J., \& Burt, K. B. (2014). Is Parenting the Mediator of Change in Behavioral Parent Training for Externalizing Problems of Youth? Clinical Psychology Review, 34(8), 608-619. doi: 10.1016/j.cpr.2014.10.001

Ginsburg, G., Grover, R., \& Ialongo, N. (2004). Parenting Behavior Among Anxious and Non-Anxious Mothers: Relation with Concurrent and Long-Term Child Outcomes. Child \& Family Behavior Therapy, 26(4), 23-41. doi: 10.1300/j019v26n04_02

Guevara, P. (2007). Factores contextuales y emociones morales como predictores de los comportamientos prosociales y problemáticos en la adolescencia. In V. Cabrera-García \& P. Guevara (Eds.), Relaciones familiares y ajuste de psicológico: dos estudios en adolescentes de familias colombianas (pp. 1-65). Bogotá: Universidad de Los Andes.

Haan, D., Prinzie, P., \& Dekoviæ, M. (2010). How and Why Children Change in Aggression and Delinquency from Childhood to Adolescence: Moderation of Over Reactive Parenting by Child Personality. Journal of
Child Psychology and Psychiatry, 51(6), 725-733. doi: 101111/j.1469-7610.2009.02192.x

Jaffe, S. R., Hanscombe, K. B., Haworth, C. M. Davis, O. S., \& Plomin, R. (2012). Chaotic Homes and Children's Disruptive Behavior: A Longitudinal CrossLagged Twin Study. Psychological Science, 23(6), 643-650. doi:10.1177/0956797611431693

Jiménez-Flores, J. (2014). Factores de riesgo familiares y conducta agresiva infantil: Un modelo ecológico de desarrollo (Tesis doctoral). Universidad Nacional Autónoma de México, México.

Jiménez-Flores, J., \& Flores-Herrera, L. M. (2014). Diseño y validación psicométrica de la Lista de Prácticas de Disciplina Severa. Revista Iberoamericana de Diagnóstico y Evaluación Psicológica, 38(2), 137-153. Recuperado de https:// www.redalyc.org/articulo.oa?id=459645434008

Kline, R. (1998). Structural equation modeling. New York: The Guilford Press.

Krahé, B., Bondü, R., Höse, A., \& Esser, G. (2014). Child Aggression as a Source and a Consequence of Parenting Stress: A Three-Wave Longitudinal Study. Journal of Research on Adolescence, 1-12. doi: 10.1111/jora.12115

Langrock, A., Compas, B., Keller, G., Merchant, J., \& Copeland, M. (2002). Coping With the Stress of Parental Depression: Parents' Report of Children's Coping, Emotional and Behavioral Problems. Journal of Clinical and Adolescent Psychology, 3(31), 312324. doi: 10.1207/S15374424JCCP3103_03

MacPhee, A, \& Andrews, J. (2006). Risk Factors for Depression in Early Adolescence. Adolescence, 163(41), 435-466.

Mercado, J. S., Landázuri, M. A., \& Terán, A. (2001). Psicología ambiental: Una visión perspectiva. In L. Flores-Herrera \& J. Bustos-Aguayo (Comps.), Concepciones en la interacción social del niño (pp. 23-77). México: Facultad de Estudios Superiores Zaragoza, UNAM.

Mora, J., Natera, G., Tiburcio, M., \& Juárez, F. (2008). Propiedades psicométricas de la Escala de Tácticas de Conflicto (CTS2) en mujeres mexicanas. Revista Mexicana de Psicología, 1(25), 107-117. 
Rasit, A., \& Songul, G. (2013). The Relationship Among Interparental Conflict, Peer, Media Effects and the Violence Behavior of Adolescents. The Mediator Role of Attitudes Toward Violence. Educational Sciences: Theory and Practice, 13(4), 2005-2015. doi: 10.1212738/estp.2013.4.1950

Reid, B. J., \& Patterson, R. G. (1989). The development of antisocial behavior patterns in childhood and adolescence. European Journal of Personality, 3, 107-119. doi: 10.1002/per.2410030205

Reppeti, R., Taylor, S., \& Seeman, T. (2002). Risky Families: Family Social Environments and the Mental and Physical Health of Offspring. Psychological Bulletin, 128(2), 330-336.

Robles, R., Varela, R., Jurado, S., \& Páez, F. (2001). Versión mexicana del Inventario de Ansiedad de Beck: propiedades psicométricas. Revista Mexicana de Psicología, 18(2), 211-218.

Sánchez, G. (2018). Factores ambientales y práctica parental en la manifestación de agresión infantil (Tesis Doctoral). Universidad Nacional Autónoma de México, México.
Sander, J., \& McCarty, C. (2005). Youth Depression in the Family Context: Familial Risk Factors and Models of Treatment. Clinical Child and Family Psychology Review, 3(8), 203-219. doi: 10.1007/s10567-0056666-3

Sauceda-García, J. M., Olivo-Gutiérrez, N., Gutiérrez, J., \& Maldonado-Durán, J. M. (2007). El castigo físico en la crianza de los hijos. Un estudio comparativo. Boletín Médico del Hospital Infantil de México, 63(6), 382-388.

Scaramella, L. V., \& Leve, L. D. (2004). Clarifying Parent-Child Reciprocities During Early Childhood: The Early Childhood Coercion Model. Clinical Child and Family Psychology Review, 2(17), 89-107. doi: 10.1023/b:ccfp.0000030287.13160.a3

Silva, A. (2003). Conducta antisocial: Un enfoque psicológico. México: Pax.

So-Kum, T. C. (2006). Corporal Punishment and Physical Maltreatment Against Children: A Community Study on Chinese Parents in Hong Kong. Child Abuse and Neglect, 30(8), 893-907. doi: 10.1016/ j.chiabu.2006.02.012

\section{Juan Jiménez Flores}

Carrera de Psicología, Facultad de Estudios Superiores Zaragoza, Universidad Nacional Autónoma de México, México

Doctor en Psicología y profesor de tiempo completo en la UNAM. El trabajo se concentra en la formación y capacitación en Psicología Clínica y Educativa; y en investigación de procesos de Psicología Familiar.

ORCID: 0000-0002-2298-7980

Autor corresponsal: jimenezj19@hotmail.com

Luz María Flores Herrera

Carrera de Psicología, Facultad de Estudios Superiores Zaragoza, Universidad Nacional Autónoma de México

Doctora en Psicología y profesora de tiempo completo en la UNAM, miembro del Sistema Nacional de Investigadores Nivel 1, su trabajo se centra en desarrollar metodología observacional y en metodología experimental.

ORCID: 0000-0002-2954-586x

lucy--_fh@yahoo.com

\section{César Merino-Soto}

Departamento de Psicología, Universidad de San Martín de Porres, Perú

Doctorante en el Centro de Investigación Transdisciplinar en Psicología de la Universidad Autónoma del Estado de Morelos. Su trabajo se centra en la medición y avances metodológicos en el ámbito de Psicología Educacional.

ORCID: 0000-0002-1407-8306

sikayax@yahoo.com.ar 